\title{
The Development of a Smart Map for Minimum "Exertion" Routing Applications
}

\author{
Katherine Carl Payne \\ Brigham Young University \\ kcpayne@byu.edu
}

\author{
Moshe Dror \\ University of Arizona \\ moshed@email.arizona.edu
}

\begin{abstract}
The problem of minimum cost routing has been extensively explored in a variety of contexts. While there is a prevalence of routing applications based on least distance, time, and related attributes, exertion-based routing has remained relatively unexplored. In particular, the network structures traditionally used to construct minimum cost paths are not suited to representing exertion or finding paths of least exertion based on road gradient. In this paper, we introduce a topographical network or "topograph" that enables minimum cost routing based on the exertion metric on each arc in a given road network as it is related to changes in road gradient. We describe an algorithm for topograph construction and present the implementation of the topograph on a road network of the state of California with $\sim 22$ million nodes.
\end{abstract}

\section{Introduction}

Exertion is an important consideration for all modes of road transportation. Vehicles require fuel to operate and release emissions based on the amount of work they perform. Constructing routes for vehicles based on minimizing emissions and fuel consumption has become a priority for network planners, policymakers, and consumers. In 2013, FedEx set a sustainability goal of $20 \%$ improvement in fuel efficiency by 2020 [4]. The United Parcel Service (UPS) reports on fuel economy in its KPIs and showed a reduction in $\mathrm{CO}_{2}$ emissions by 13,000 metric tonnes from 2012 to 2013 [25]. Exertion is also important to commuting bicyclists and pedestrians, as they exert physical effort and burn calories based on the strenuousness of their exercise. There has been a surge in the production of devices and applications to track road data and indicators of exertion during running trips and bicycle commutes. MapMyFitness, an application that allows users to integrate road data from their walks, running, bicycle riding, and hiking with exertion indicators has over 9 million active users [23].
While applications exist that construct routes of minimal exertion for vehicles, bicycle riders, and pedestrians, they are at core based on minimizing distance and time, as they select arcs of minimal distance and time costs to generate routes. A recent study of vehicle fuel economy demonstrated that traditional energy consumption models have neglected road gradient as a key factor and, as a result, actually produce routes of greater energy consumption when they do not account for road gradient [17]. In addition, the major determining factor of the exertion of a bicycle rider on a given route is variability in road gradient [3]. In order to make road gradient the primary attribute of concern when constructing routes of minimal exertion (fuel consumption or physical effort), a new network structure is needed, as the traditional road network can only be used to construct routes of minimal distance and time.

In this paper, we introduce a topographical network or topograph that enables minimum exertion routing based on the exertion metric for each arc as it is related to road gradient. Previous work related to fuel consumption and human exertion is discussed in Section 2. The method used to transform a traditional road network into a topograph is presented in Section 3 . The results of topograph construction for the entire state of California are described in Section 4. Application of the topograph to minimal exertionbased routing is discussed in Section 5 and conclusions are presented in Section 6.

\section{Related Work}

Road gradient is a key component in exertionbased routing for road transportation. We review network structures used for exertion-based routing methods for vehicles, bicyclists, and pedestrians. Many of the existing methods do not incorporate road gradient as a factor and those that do so only use average or maximum elevation along arcs weighted by distance and time to construct routes. 


\subsection{Vehicle Fuel Economy}

Several systems and methods have been proposed for constructing routes of minimal fuel consumption. Fuel consumption has traditionally been calculated as a function of the power requirements needed to overcome aerodynamic resistance, rolling resistance, gravitational resistance, and gravitational potential energy [17]. Methods for constructing routes of minimal fuel consumption often consider that fuel consumption varies linearly with a vehicle's weight per unit distance [7],[27]. A study of eco-routing algorithms quantifying the impact of implementing a system in Ohio used fuel consumption to construct optimal routes [9]. Route-first cluster-second techniques have also been used to develop systems that produce routes of minimal fuel consumption [11]. While they consider fuel consumption in route construction, these systems use a traditional road network with arc weights of distance and time to find routes of minimal fuel consumption.

\subsection{Bicycles and Pedestrians}

There are several systems available that generate bicycle and pedestrian routes, some of which can be used to construct routes of minimal exertion. A route planner for Broward County, FL described is built with the ArcGIS JavaScript Extension for Google Maps API and the Google Local Search Control for Maps API [10]. The system uses the ArcGIS Network Analyst extension to search for the fastest, simplest, most scenic, and shortest routes based on the maps provided by Google. Similarly, a planner for Vancouver is a Google Maps mashup application that allows cyclists to generate routes based on preferences of distance, elevation gain, air quality, and scenery [12]. A bicycle route planner for Charlottesville, VA also uses the Google Maps API to generate routes based on user preferences of safety, difficulty, and distance [24]. The ROSE system uses pedestrian navigation and public transportation recommendations to guide users to locations and events of interest [2]. A walking recommender system has also been proposed that considers route safety, amenity, and walkability [26]. While some of these systems consider changes in road gradient, they use a traditional road network with arc weights of distance and time to find routes of minimal exertion.

\subsection{Road Network Representation}

Routing applications require the use of graphs to represent road network structure. The traditional representation of a road map uses nodes in the graph to represent road intersections and edges or arcs to represent the sections of road linking intersections. Several well-known network flow problems that use graph representations include the shortest path, maximum flow, minimum cost flow, and multicommodity flow problems [1]. Graph representations of roads have been used in GIS-based routing for a variety of applications, including choosing optimal roads to reach timber stands in forests and routing the collection of solid waste in India by Ghose et al. [6],[18]. Extensions and manipulations to the traditional road graph model have been made in GIS applications in order to capture road topology. For example, Speičys and Jensen developed a graph representation of a road network that combines a two-dimensional representation of roads with a multi-graph representation that simultaneously captures the details of road features and allows for routing [16]. Wilke et al. created a lane-centric graph representation of roads to produce large-scale traffic simulations [5].

While the traditional road network facilitates routing based on least distance and time, it is not designed to facilitate minimal exertion routing as it is related to road gradient. To meet the need for a structure that enables minimal exertion routing based on road gradient, we define a topograph.

\section{Topograph Construction Method}

We use a traditional road network as input to the transformation algorithm to construct a topograph. A traditional road network is defined as a directed graph $G=(V, A)$ where the set $V$ is a finite set of nodes in which every $v \in V$ represents a point on a road and the set $A$ is a finite set of arcs where every $\operatorname{arc} a=(v, u) \in A$ connects two nodes $u$ and $v$ from $u$ to $v$. In contrast, a topograph is defined as a directed $\operatorname{graph} \widetilde{G}=(\tilde{V}, \tilde{A})$ in which every $v \in \tilde{V}$ represents a point on a road at which there is a change in incline or an intersection and the set $\tilde{A}$ is a finite set of arcs where every arc $a=(v, u) \in \tilde{A}$ connects two nodes $u$ and $v$ and is of constant change in incline throughout. In particular, an arc in a traditional road network may have several places at which the road gradient changes. In a topograph, changes in road gradient only occur at nodes $v \in \tilde{V}$.

We start the transformation of $G$ to the topograph

$\tilde{G}$ at a node $v$ in the digital road network and traverse it along an incident $\operatorname{arc}(v, u)$ until we reach either (1) 
a point $p$ at which the percent grade incline changes, or (2) node $u$. Each time the percent grade incline changes along $\operatorname{arc}(v, u)$ the point at which it changes $p$ is added and appropriately labeled as a node in the topograph, and the arc incident to it $(v, p)$ is added as an arc in the topograph. In this manner, an arc from the traditional road network $G$ is replaced by a set of successive arcs, each with constant change in incline throughout. For example, an arc in the traditional road network may be comprised of three sections of $0 \%, 1 \%$, and $-1 \%$ road gradient. In the topograph, this arc will be replaced by three separate successive arcs of $0 \%, 1 \%$, and $-1 \%$ road gradient. When an intersection in the digital road network is reached, a corresponding node and incident arc is also added to the topograph. Nodes are added at where a vehicle or person is required to stop. Arcs will only be added (and appropriately labeled) to the topograph if they begin and end at points at which the percent grade incline changes or if they are representative of an intersection. Traversal of the digital road network $G$ and its transformation into the corresponding topograph $\tilde{G}$ terminates when all nodes in $G$ have been visited.

\section{Results}

We executed the topograph construction on the road network of the state of California and produced a topograph of 22,238,152 nodes. Visualization of the new structure is shown in Figure 1.

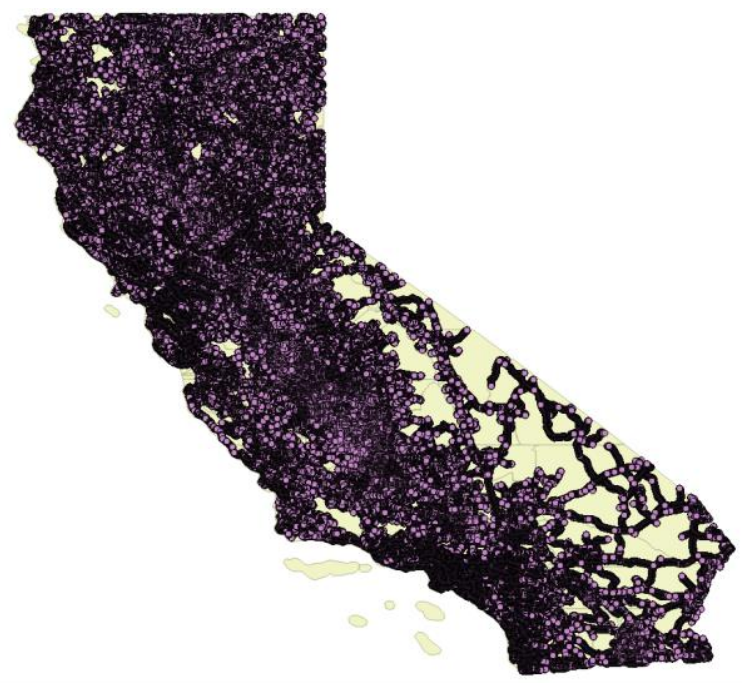

Figure 1: Topograph of California

\subsection{Data Integration}

We used the open source OpenStreetMap (OSM) dataset as input to construct a topograph of California [20]. Preprocessing was performed on the data to create the input graph $G$ with elevation attributes. The OSM dataset model is comprised of three basic elements, including nodes, ways, and relations. Node elements are used to represent point features, such as points of interest or points that define the shape or path of a way. Ways are sets of up to 2,000 nodes that represent linear features such as roads, streams, and railway lines. Relations are defined as ordered lists of one or more nodes and/or ways that are used to model geographical relationships between objects. We used the OSM ways representative of roads in California and the nodes within them to create our input graph $G$.

To find segments of constant incline throughout, we used the 1/3-arc second Digital Elevation Model (DEM) available from the U.S. Geological Survey that provides elevation data at a resolution of 10 meters [19]. A PostgreSQL database with PostGIS extensions was used to store the OSM road network and corresponding elevation data retrieved with Python scripts. Each way in the OSM road network is stored with a corresponding collection of nodes that illustrate the shape of the way. For example, part of Portola Road is shown as a collection of four nodes in Figure 2.

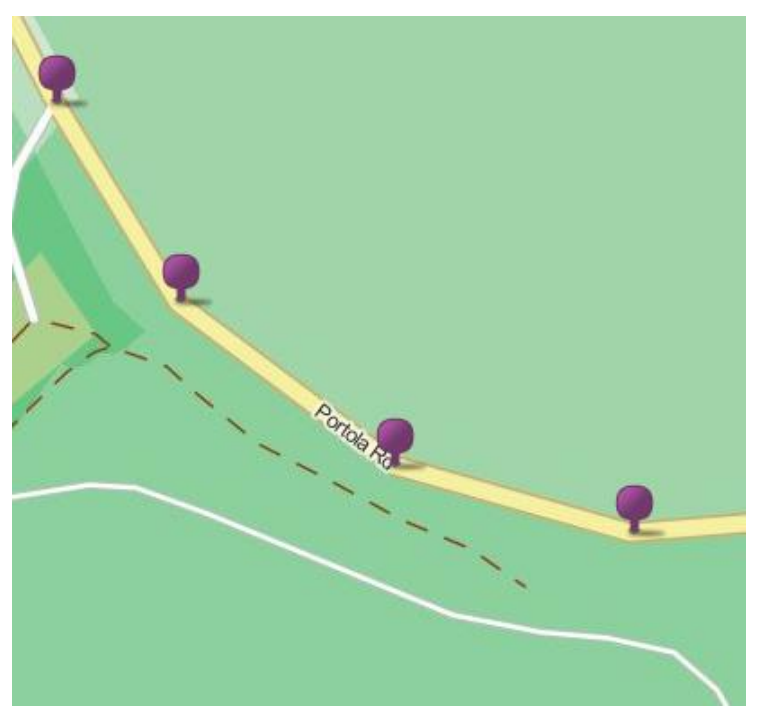

Figure 2: Portola Road OSM

In order to obtain the highest level of elevation granularity related to the DEM during topograph construction, we first redefined each way as a collection of nodes 10 meters apart using navigation formulas. The results of this preprocessing for 
Portola road are shown in Figure 3. If an original node in the way is an intersection, we include it in the new collection of nodes. The effects of intersection inclusion can be seen in the upper left portion of Portola road, where there are nodes 10 meters apart and an intersection between them. During processing we defined an intersection as a node that belonged to two or more ways. This includes nodes that may not be intersections of two roads, but the intersection of two ways that represent the same road.

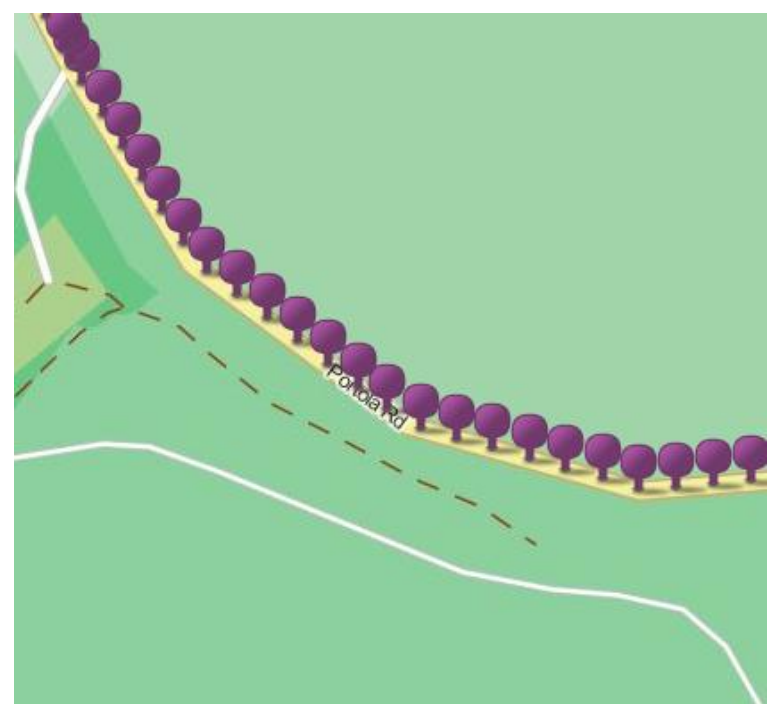

Figure 3: Portola Road with 10m Nodes

Once all ways were redefined as collections of nodes 10 meters apart, we assigned elevation data to each node from the DEM by querying the DEM for elevation at each node's geographic coordinates. Using the elevation data, road gradient was calculated for every arc in $A$. This assignment produced the input graph $G=(V, A)$ where each OSM node with elevation data is a node $v \in V$ and every adjacent node pair defines an $\operatorname{arc}(v, u) \in A$ with a road gradient assignment.

\subsection{Algorithm Implementation}

The entire state of California took six days of processing time to complete. The algorithm began processing at a node representative of a dead end in Williams, CA. The traversal of the road network proceeded as described in Section 3 using a depthfirst search procedure to process every node in the graph. Two PostgreSQL tables served as input and output, respectively. Each table represented an adjacency list of nodes. The input table contained the nodes $10 \mathrm{~m}$ apart in $G$ and the output table contained the adjacency list of nodes in the topograph $\tilde{G}$.
Additional information was retained in the topograph table to keep track of the original nodes belonging to each new arc in $\tilde{A}$. A street-level view of the resultant topograph is shown in Figure 4. Every node in the topograph shown represents a change in the road gradient. Arcs in the topograph connecting pairs of nodes $(v, u) \in \tilde{A}$ are segments of road with constant change in road gradient throughout.

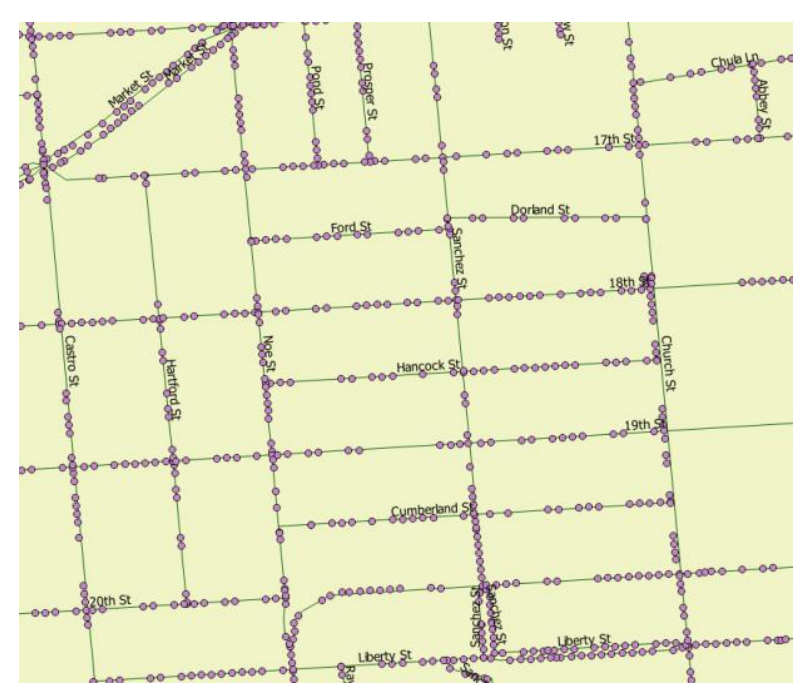

Figure 4: Street View of Topograph

\subsection{Data Limitations}

While we have chosen to use the DEM provided by the USGS and the road network data provided by OSM to illustrate the graph transformation method, any elevation and road network data can be used for this purpose. These data sets, like others available, are not without limitations. The 30-meter resolution elevation model has been shown to have large local error values, although the average error in the data set is small [15]. Determining elevation in urban areas is challenging and there is a need for algorithms that filter out vegetation and capture locally significant land features [13]. In addition, the raster format of DEMs reduces the accuracy of slope calculations [22]. An investigation for the Florida Department of Transportation demonstrated that it would be possible to implement a multimodal trip planner using USGS DEMs and OSM data, although data coverage remains incomplete [8]. In some cases, however, OSM data is more complete than proprietary data sets [21]. Despite its limitations, the data in OSM is still relatively high. We have chosen the USGS DEM and OSM data to illustrate our graph transformation for the range of their coverage in the United States, as well as their high levels of granularity. 


\section{Routing Applications}

Once constructed, a topograph can be used to construct routes of minimal exertion for vehicles, bicycles, and pedestrians based on exertion as it is related to road gradient. For example, the model of perceived exertion developed by Carl et al. can be applied to the topograph to construct routes of minimal perceived exertion for touring bicyclists [14]. Their model calculates perceived exertion for cyclists of different levels of expertise based on the gradient of the road. Because all of the arcs in the topograph are of constant change in incline throughout, weights of exertion can be assigned to them (similar to values of distance or time in a traditional graph) to facilitate minimal cost routing. Similarly, the topograph can be used to construct routes of minimal fuel consumption based on road gradient. Minimum energy routes calculated using changes in road gradient are more accurate and better suited to routing based on fuel consumption than those that rely on changes in speed, acceleration, and braking alone [17]. A model for fuel economy based on road gradient at its core while incorporating other factors can be used to calculate fuel consumption and assign appropriate weights to the topograph to construct routes of minimal exertion. Whether determining routes for vehicles, bicyclists, or pedestrians, the topograph provides the underlying structure needed to apply models of exertion and generate minimum cost routes based on exertion that a traditional road network does not.

\section{Conclusion}

In this paper, we presented a method of transforming a traditional road network into a topograph suited for exertion-based routing using polyline data from the OSM and elevation data from a DEM provided by the U.S. Geological Survey. Unlike a traditional road network, the topograph has nodes at every point at which there is a change in incline in the road and every arc in the graph has constant change in incline throughout. While a traditional road network can be used to solve routing problems such as the shortest path problem, its structure cannot be used to generate routes of minimal exertion. The graph transformation described in this paper provides a foundation for applications that generates minimum cost routes based on exertion. Because it provides a unique representation of road topography that can be applied to various measures of effort, the topograph can serve as a structure for minimal exertion-based routing in applications in logistics, personal health, and defense.

\section{References}

[1] Ahuja, R.K., Magnanti, T.L., and J.B. Orlin, Network Flows: Theory, Algorithms and Applications, Prentice Hall, Upper Saddle River, NJ, 1993.

[2] B. Ludwig, B. Zenker, and J. Schrader, "Recommendation of Personalized Routes with Public Transport Connections," Intelligent Interactive Assistance and Mobile Multimedia Computing Communications in Computer and Information Science, 53 (2009), pp. 97-107.

[3] Burke, E.R., High-tech Cycling, 2nd Ed., Human Kinetics, Champaign, IL, 2003.

[4] C. Wolski, "FedEx Sets New Environmental Goal," Green Fleet Magazine, 2013. Retrieved from http://www.greenfleetmagazine.com/article/51762/fed ex-sets-new-environmental-goal/.

[5] D. Wilkie, J. Sewall, and M.C. Lin, "Transforming GIS Data into Functional Road Models for LargeScale Traffic Simulation," IEEE Transactions on Visualization and Computer Graphics, 18 (2012), pp. 890-901.

[6] D.J. Dean, "Finding Optimal Routes for Networks of Harvest Site Roads Using GIS-based Techniques," Canadian Journal of Forest Research, 27 (1997), pp. 11-22.

[7] D.R. Gaur, A. Mudgal, and R.R. Singh, "Routing Vehicles to Minimize Fuel Consumption," Operations Research Letters, 41 (2013), pp. 576-580.

[8] E. L. Hillsman and S.J. Barbeau, "Enabling CostEffective Multimodal Trip Planners through Open Transit Data," (FDOT BDK85 \#977-20) National Center for Transit Research, University of South Florida, 2011.

[9] H. Rakha and K. Ahn, "Developing an Eco-Routing Application,” (DTRT12-G-UTC17) U.S. Department of Transportation, Virginia Polytechnic Institute and State University, 2014.

[10] H.H. Hochmair and J. Fu, "Web Based Bicycle Trip Planning for Broward County, Florida," ESRI User Conference, San Diego, CA, USA, 2009.

[11] J.A. Montoya, C. Guéret, J.E. Mendoza, and J.G. Villegas, "A Route-First Cluster-Second Heuristic for the Green Vehicle Routing Problem," ROADEF15ème congrès annuel de la Société française de recherche opérationnelle et d'aide à la decision, Bordeaux, France, 2014.

[12] J.G. Su, M. Winters, M. Nunes, and M. Brauer, "Designing a Route Planner to Facilitate and Promote Cycling in Metro Vancouver, Canada," Transportation Research Part A, 44 (2010), pp. 495505.

[13] J.P. Wilson, "Digital Terrain Modeling," Geomorphology, 137 (2012), pp. 107-121.

[14] K. Carl, S.A. Brown, M. Dror, and A. Durcikova, "Bicycle Tours: Modeling the Perceived Exertion of a Path," Journal of Quantitative Analysis in Sports, 9 (2013), pp. 203-216.

[15] K.W. Holmes, O.A. Chadwick, and P.C. Kyriakidis, "Error in a USGS 30-Meter Digital Elevation Model 
and its Impact on Terrain Modeling," Journal of Hydrology, 233 (2000), pp. 154-173.

[16] L. Speičys and C.S. Jensen, "Enabling Location-based Services-Multi-Graph Representation of Transportation Networks," GeoInformatica, 12 (2008), pp. 219-253.

[17] M. Duell, M.W. Levin, and S.T. Waller, "The Impact of Road Grade on City-Wide Vehicle Energy Consumption and Eco-routing," Proceedings of the $32^{\text {nd }}$ Conference of Australian Institutes of Transport Research (CAITR). University of New South Wales, Sydney, 2014.

[18] M.K. Ghose, A.K. Dikshit, and S.K. Sharma, "A GIS based Transportation Model for Solid Waste Disposal-A Case Study on Asansol Municipality, Waste Management, 26 (2006), pp. 1287-1293.

[19] National Elevation Dataset, U.S. Geological Survey, 2014. Retrieved from http://ned.usgs.gov/.

[20] OpenStreetMap, 2013. Retrieved from http://www.openstreetmap.org/.

[21] P. Neis, D. Zielstra, and A. Zipf, "The Street Network Evolution of Crowdsourced Maps: OpenStreetMap in Germany 2007-2011," Future Internet, 4 (2011), pp. $1-21$.

[22] Q. Zhou and X. Liu, "Analysis of Errors of Derived Slope and Aspect Related to DEM Data Properties," Computers and Geosciences, 30 (2004), pp. 369-378.

[23] R. Empson, "Under Armour Buys Mobile Workout Veteran MapMyFitness for $\$ 150 \mathrm{M}$ as it looks to Keep Pace in Digital Fitness," Tech Crunch, 2013. Retrieved from http://techcrunch.com/2013/11/14/under-armour-putsdown-150m-to-acquire-fitness-tracking-platformmaymyfitness-and-its-20m-users/.

[24] R.J. Turverey, D.D. Cheng, O.N. Blair, J.T. Roth, G.M. Lamp, and R.C. Cogill,. Charlottesville Bike Route Planner. Proceedings of the IEEE Systems and Information Engineering Design Symposium, Charlottesville, VA, USA, 2010.

[25] "UPS Sustainability Report Details Fuel Use Reduction of 1.3 Million Gallons in 2012," Automotive Fleet. Retrieved from http://www.automotive-fleet.com/channel/fuelmanagement/news/story/2013/07/ups-sustainabilityreport-details-savings-of-1-3-million-gallons-of-fuelin-2012.aspx/.

[26] W. Sasaki, "Walking Route Recommender System Considering SAW Criteria," Proceedings of the 2013 Conference on Technologies and Applications of Artificial Intelligence (TAAI), 2013.

[27] Y. Xiao, Q. Zhao, I. Kaku, and Y. Xu, "Development of a Fuel Consumption Optimization Model for the Capacitated Vehicle Routing Problem," Computers and Operations Research, 39 (2012), pp. 1419-1431. 